\title{
The Kilonova Data Challenge
}

\author{
Marcelo Avila | Benedictine University
}

\section{Introduction}

The Vera Rubin Observatory Legacy Survey of Space and Time (LSST) is a project using a dedicated 8.4-meter telescope and 3.2 gigapixel camera to conduct a survey of the southern sky. We are investigating how well LSST will be able to find kilonovae by creating the "Kilonova Data Challenge." Kilonovae are cosmic events where two neutron stars or a neutron star and a black hole collide. The first way we detect these events is through gravitational waves. Gravitational waves occur when massive objects accelerate, producing ripples in spacetime. Kilonovae are rare events (somewhere between 1 to 10 every year) so being able to simulate them is extremely helpful. Data challenges are high fidelity simulations of large numbers of real astrophysical objects. We are completing this project within the LSST Dark Energy Science Collaboration (DESC) following the DESC second data challenge (DC2).

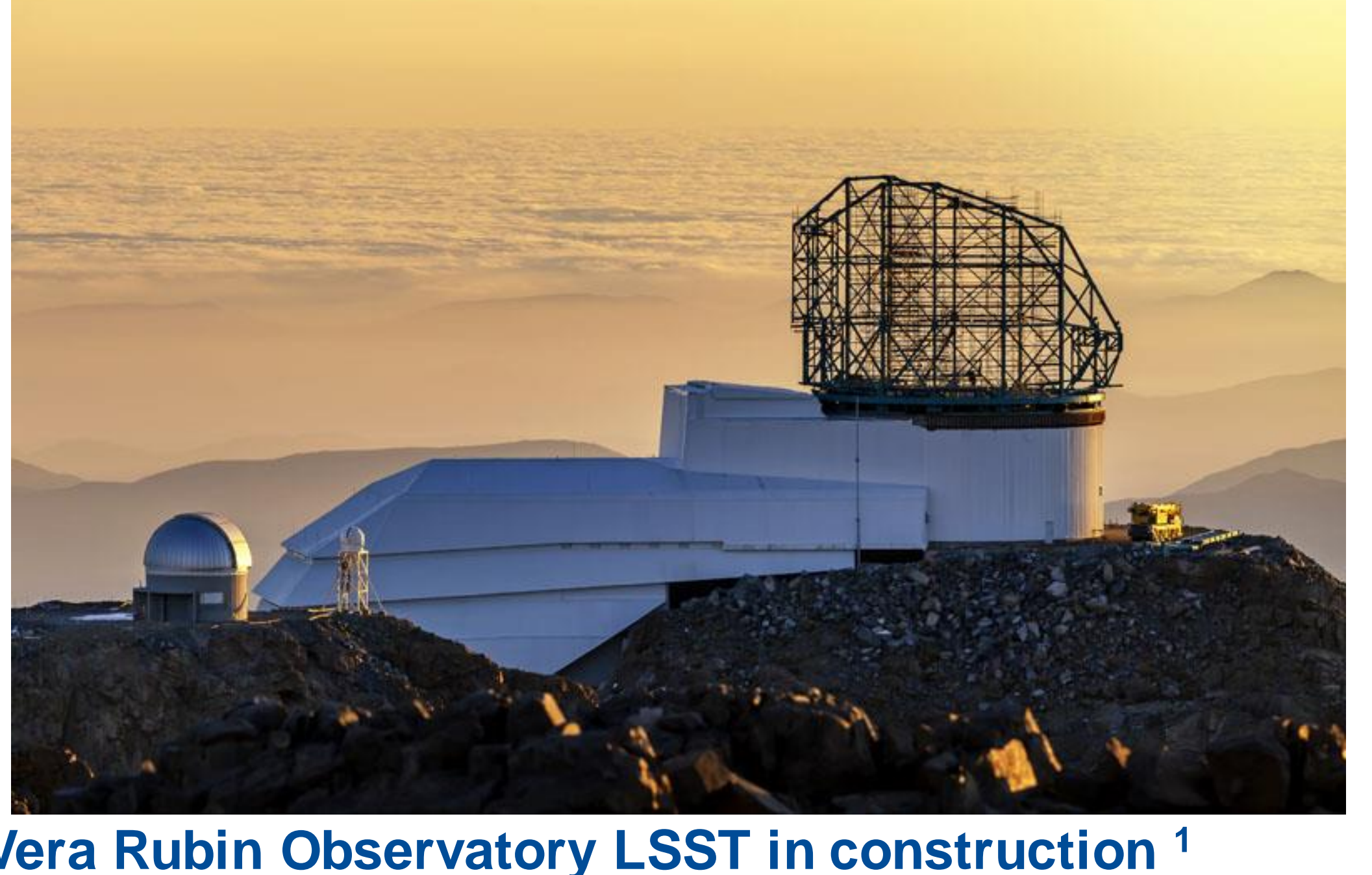

Objectives

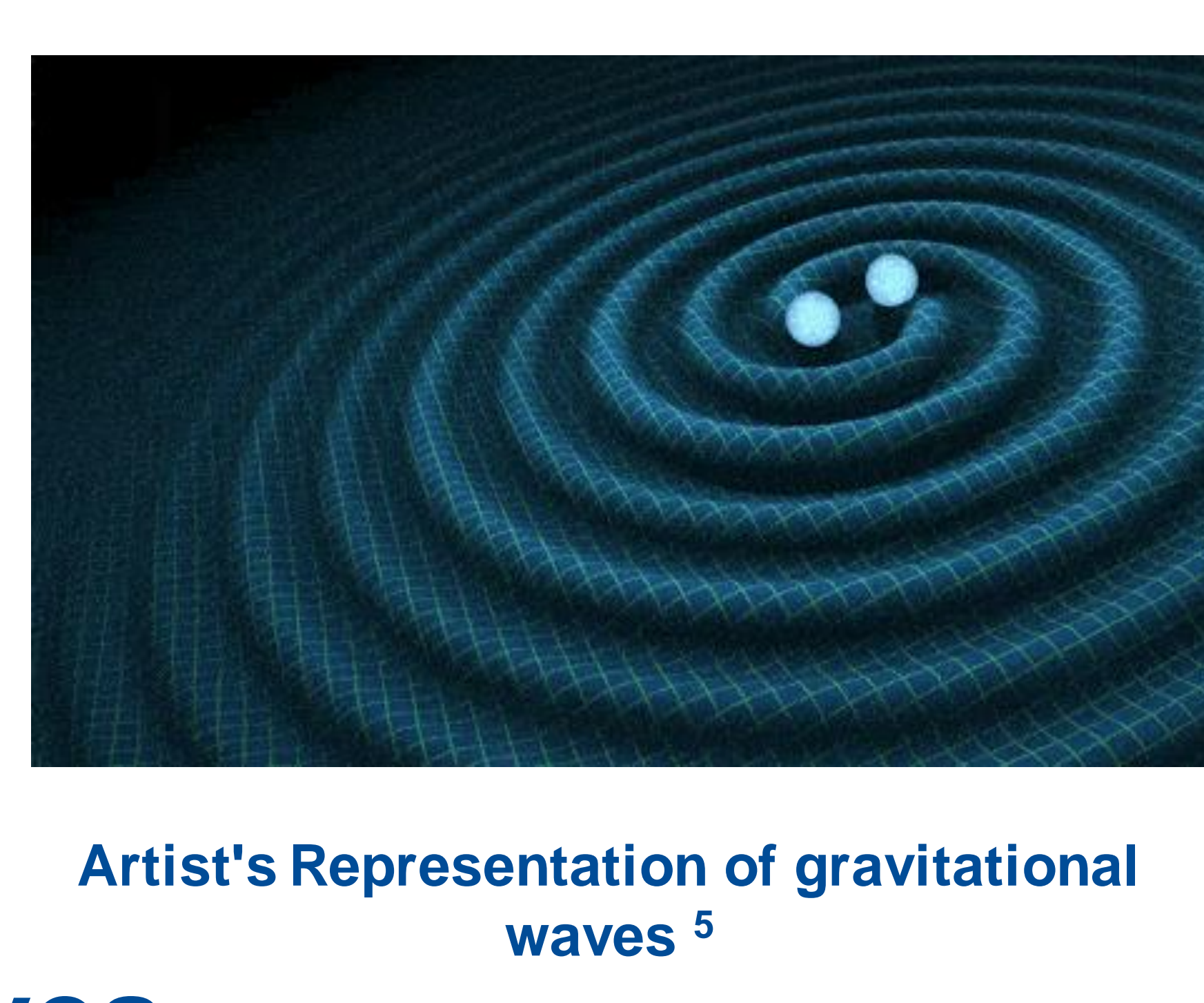

The question we set out to answer is: How well can we find kilonovae in LSST? In order to answer this our current objective is to simulate 1 square degree of sky that is overpopulated with kilonovae. We plan to use a small subset of DC2 catalogs in order to produce these simulated images.

Acknowledgements

This manuscript has been authored by Fermi Research Alliance, LLC under Contract No. DE-AC02-07CH11359 with the U.S. Department of Energy, Office of Science, Office of High Energy Physics.

\section{Methods}

In order to create these simulations, we use a program called PhoSim ${ }^{2}$. PhoSim can simulate a range of astrophysical objects; we use a point source to simulate kilonovae. To produce the spectral energy distributions (SEDs) and input object magnitudes needed by PhoSim we implemented Kasen models ${ }^{3}$ using a synthetic photometry code ${ }^{4}$. Below we show the SEDs produced by the code and a kilonova varying in time produced by PhoSim.
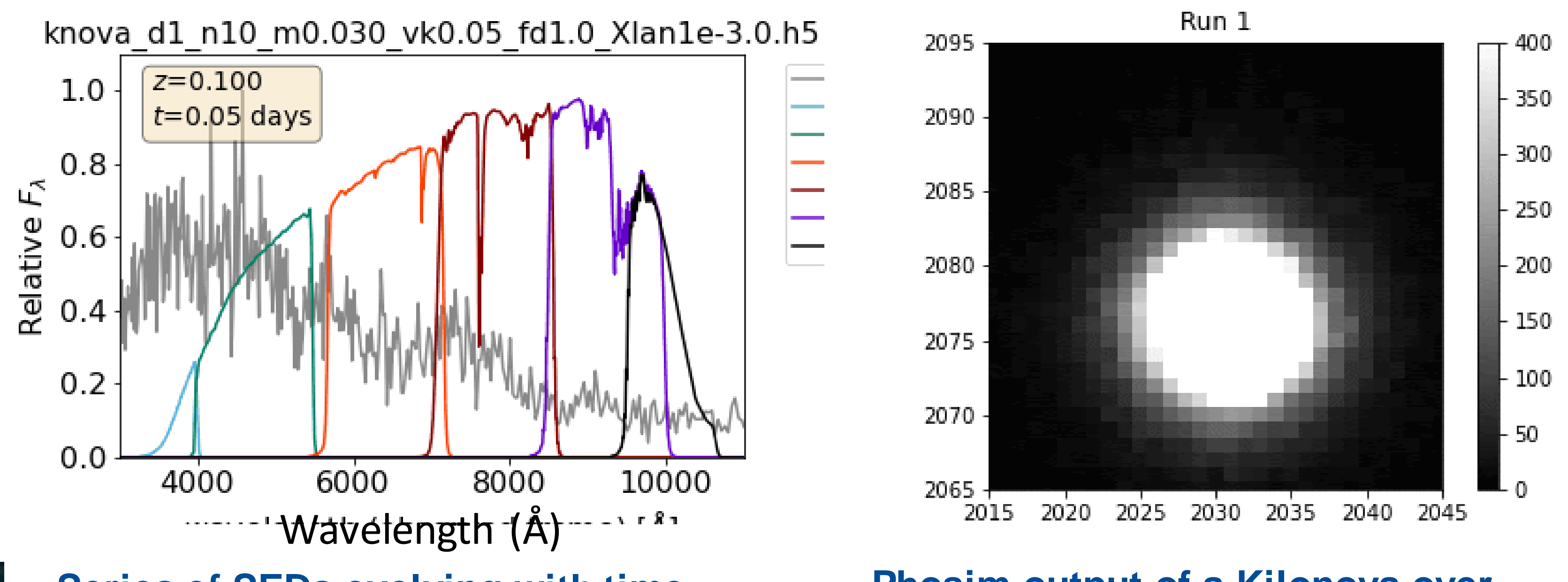

Series of SEDs evolving with tim showing the evolution of the spectrum of the amount of light
being produced over time with Dark Energy Survey filter curves

With this we can then input the kilonovae into DC2 images producing a realistic scene of a kilonova with a host galaxy. To do this we also simulate a DC2 image and add the information for the kilonova to the catalog. This simulates both the kilonova and the rest of the objects. Here is the image of a kilonova in a DC2 image. It is in the center of the right image. The left is a zoomed in version where the kilonova is the green object.
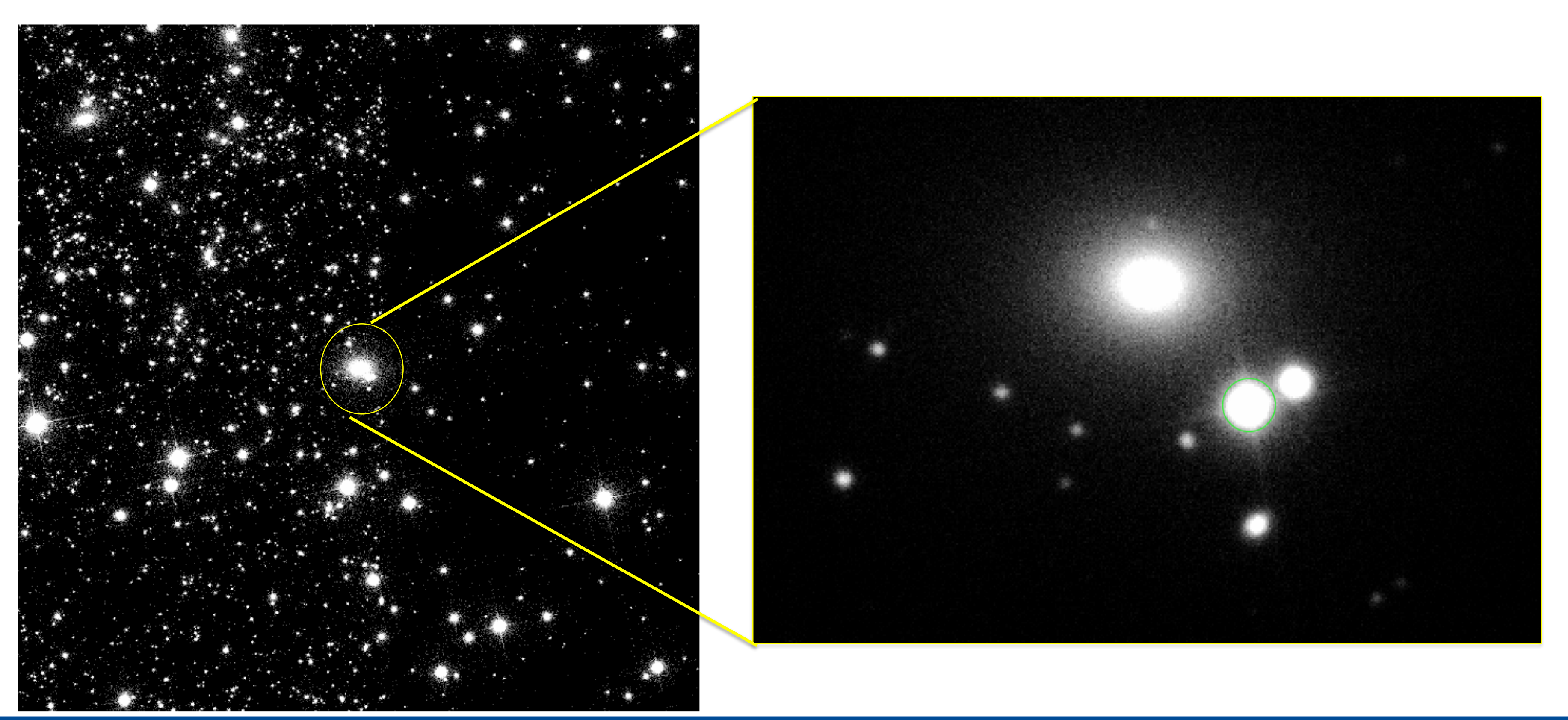

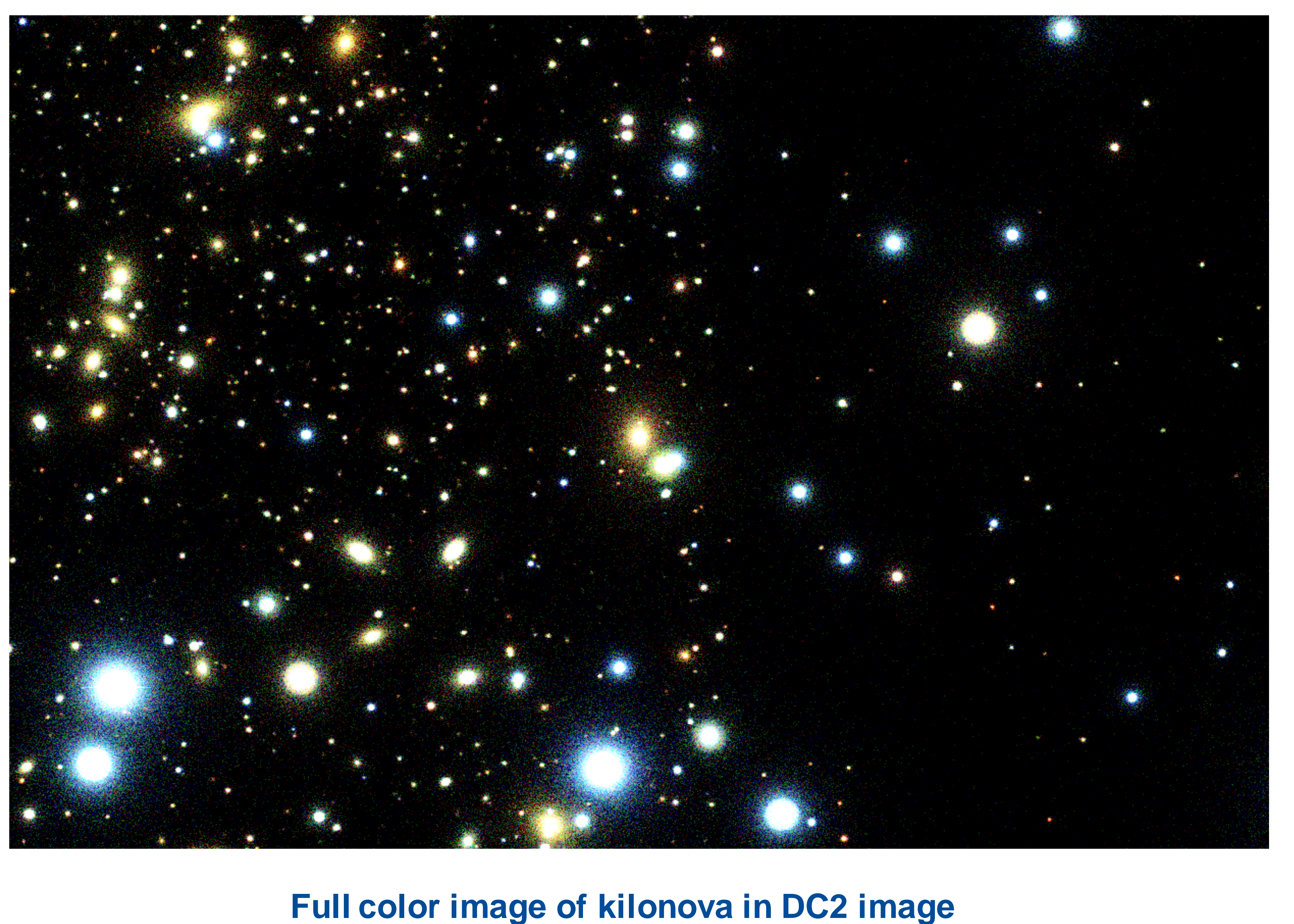

Conclusion/Summary

These images are useful because they will help test the GW kilonova-finding pipelines in preparation for the beginning of LSST operations in 2023.

\section{Future Directions}

In the future we plan to simulate 25 square degrees instead of just 1 . These 25 square degrees will also be overpopulated with kilonovae. To do this we will continue using PhoSim to produce these images and we will try using a source injection method where we use synthetic source injection to put out kilonovae into a real DC2 image without having to simulate every step. Finally, after we have all our images and catalogs, we will test the GW finding pipelines to see how well they are able to identify the kilonovae we have added to the images.

\section{Resources}

1: "Vera Rubin Observatory's Legacy Survey of Space and Time: Kavil Institute for Particle Astrophysics and Cosmology (KIPAC)." Vera Rubin Observatory's Legacy Survey of Space (K)

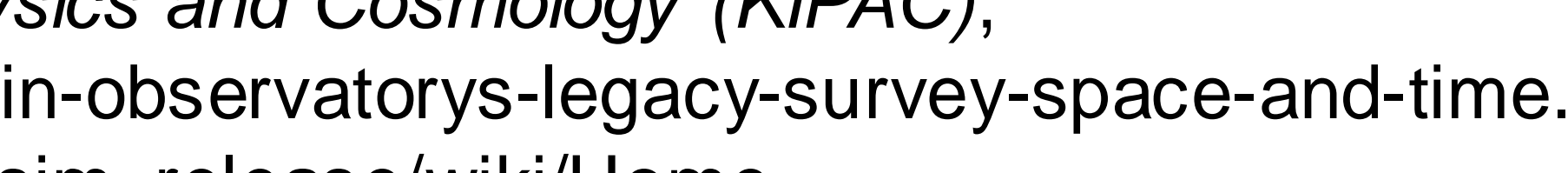
3: Kasen, Daniel, and S. E. Woosley. "Type li Supernovae: Model Light Curves and Standard Candle Relationships." NASA/ADS, ui.adsabs.harvard.edu/abs/2009ApJ...703.2205K/abstrac . 4: https://github.com/douglasleetucker 